Ther modynami cs of the sel f-gravi tat ing ring model

\begin{tabular}{|l|l|}
\hline 著者 & $\begin{array}{l}\text { TATEKAMA Takayuki , BOUCHET Fr eddy, DAUXO S } \\
\text { Thi er ry, RUFFO St ef ano }\end{array}$ \\
\hline $\begin{array}{l}\text { j our nal or } \\
\text { publ i cat i on t i t l e }\end{array}$ & Physi cal revi ew. Thi $r$ d ser i es. E \\
\hline vol une & 71 \\
\hline page range & 56111 \\
\hline year & $2005-05$ \\
\hline URL & ht t p: //hdl . handl e. net /10098/6417 \\
\hline
\end{tabular}




\title{
Thermodynamics of the self-gravitating ring model
}

\author{
Takayuki Tatekawa, ${ }^{1,2}$ Freddy Bouchet, ${ }^{1}$ Thierry Dauxois, ${ }^{1}$ and Stefano Ruffo ${ }^{1,3}$ \\ ${ }^{1}$ Laboratoire de Physique, UMR-CNRS 5672, ENS Lyon, 46 Allée d'Italie, 69364 Lyon cédex 07, France \\ ${ }^{2}$ Department of Physics, Waseda University, 3-4-1 Okubo, Shinjuku, Tokyo 169-8555, Japan \\ ${ }^{3}$ Dipartimento di Energetica, "S. Stecco" and CSDC, Università di Firenze, and INFN, via S. Marta, 3, 50139 Firenze, Italy
}

(Received 25 January 2005; published 19 May 2005)

\begin{abstract}
We present the phase diagram, in both the microcanonical and the canonical ensemble, of the selfgravitating-ring (SGR) model, which describes the motion of equal point masses constrained on a ring and subject to 3D gravitational attraction. If the interaction is regularized at short distances by the introduction of a softening parameter, a global entropy maximum always exists, and thermodynamics is well defined in the mean-field limit. However, ensembles are not equivalent and a phase of negative specific heat in the microcanonical ensemble appears in a wide intermediate energy region, if the softening parameter is small enough. The phase transition changes from second to first order at a tricritical point, whose location is not the same in the two ensembles. All these features make of the SGR model the best prototype of a self-gravitating system in one dimension. In order to obtain the stable stationary mass distribution, we apply an iterative method, inspired by a previous one used in 2D turbulence, which ensures entropy increase and, hence, convergence towards an equilibrium state.
\end{abstract}

DOI: 10.1103/PhysRevE.71.056111

PACS number(s): 05.70.Fh, 05.20.-y, 98.10.+z

\section{INTRODUCTION}

There are many objects in our universe whose behavior can be understood considering only the gravitational interaction. Examples are globular clusters, galaxies, clusters of galaxies, molecular clouds [1]. Different theoretical approaches have been proposed to explain the peculiar statistical properties of self-gravitating systems. The main difficulty is that these systems cannot approach statistical equilibrium because of the short-distance divergence of the potential and of the evaporation at the boundaries. Even if one puts the system in a box with adiabatic walls, thus eliminating evaporation, still gravity causes the well-known phenomenon of $\mathrm{gra}$ vothermal catastrophe [2-4]. The introduction of a smallscale softening of the interaction potential, as for instance in self-gravitating fermions [5-7], avoids such a catastrophe, so that self-gravitating systems can approach the final (thermal) equilibrium state. However, such a state may have a negative specific heat. Moreover, a first order phase transition from the high energy gas phase to the low energy clustered phase appears [3].

Direct studies of the full three-dimensional $\mathrm{N}$-body gravitational dynamics are particularly heavy [8] and even special purpose machines have been built to this aim [9]. Therefore, lower dimensional models have been introduced to describe gravitational systems with additional symmetries. For instance, the gravitational sheet model describing the motion of infinite planar mass distributions perpendicularly to their surface has been considered [10]. Although this model shows interesting behaviors $[11,12]$, the specific heat is always positive and no phase transition is present. Other onedimensional self-gravitating toy models may have phase transitions and ensemble inequivalence [13].

Recently, another one-dimensional model has been introduced [14] where particle motion is confined on a ring, but the interaction is the true Newtonian $3 \mathrm{D}$ one. At short distances, the potential is regularized, so that the particles do not interact. This model has been called the self-gravitating ring model (SGR) and will be the subject of the study discussed in this paper. It has been shown in numerical simulations [14], that this model maintains the peculiar features of the 3D Newtonian potential, showing a negative specific heat phase and a phase transition if the softening parameter is small enough. Moreover, for large softening, this model reduces to the Hamiltonian mean-field model (HMF) [15], which has been recently extensively studied as a prototype system with long-range interactions. This latter model, however, although it displays a second order phase transition, does not have a negative specific heat phase at equilibrium.

In this paper, we derive the equilibrium thermodynamics of the SGR model both in the canonical and in the microcanonical ensemble. For all nonvanishing softening parameter values, this model has a thermal equilibrium state. If the softening parameter is small enough, the model shows ensemble inequivalence $[16,17]$ with a negative specific heat phase in the microcanonical ensemble and a first order phase transition. Therefore, the SGR model displays several features of the true 3D Newtonian interaction, and can serve as a better prototype of self-gravitating systems in one dimension than all previously introduced models.

The paper is organized as follows. In Sec. II, we briefly introduce the SGR model and we discuss the essential features of previous numerical simulations [14]. In Sec. III, we show the general scheme for deriving all stationary density distributions which maximize Boltzmann-Gibbs entropy at fixed total energy and mass. Section IV presents an iterative method which ensures entropy increase and leads in a unique way towards the stable equilibrium single particle distribution function. The method is inspired by a similar one used to compute entropy maxima in 2D turbulence [18]. In Sec. V, we describe in full detail how to implement the iterative algorithm in a numerical scheme. In Sec. VI, we calculate the thermodynamic quantities of the SGR model using the iterative method. We also show how, reducing the softening 


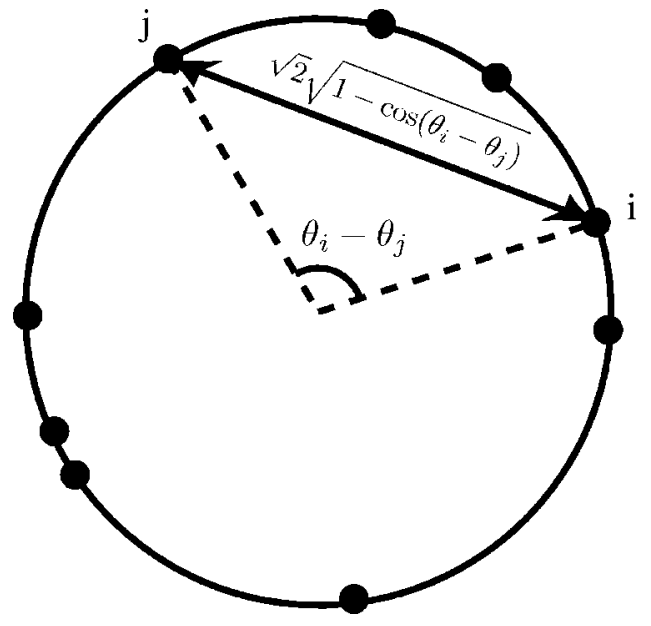

FIG. 1. Self-gravitating ring model with a fixed unitary radius. Particles are constrained to move on a ring and therefore their location is specified by the angles measured with respect to a fixed direction. Each pair of particles at $\theta_{i}$ and $\theta_{j}$ interacts through the inverse-square three-dimensional gravitational force. The distance is measured by the chord, as shown in the figure.

parameter, one enters into a region of ensemble inequivalence, where a tricritical point exists which is not the same in the two ensembles [19]. Finally, in Sec. VII, we discuss the dynamical evolution of the SGR model, emphasizing the properties of relaxation to equilibrium.

\section{SELF-GRAVITATING RING MODEL}

In this section, we briefly present the self-gravitating ring (SGR) model [14]. In this model, particle motion is constrained on a ring and particles interact via a true 3D Newtonian potential (Fig. 1).

The Hamiltonian of the SGR model is

$$
\begin{gathered}
H=\frac{1}{2} \sum_{i=1}^{N} p_{i}^{2}+\frac{1}{2 N} \sum_{i, j} V_{\varepsilon}\left(\theta_{i}-\theta_{j}\right), \\
V_{\varepsilon}\left(\theta_{i}-\theta_{j}\right)=-\frac{1}{\sqrt{2}} \frac{1}{\sqrt{1-\cos \left(\theta_{i}-\theta_{j}\right)+\varepsilon}},
\end{gathered}
$$

where $\varepsilon$ is the softening parameter, which is introduced, as usual, in order to avoid the divergence of the potential at short distances.

Taking the large $\varepsilon$ limit, the potential becomes

$$
V_{\varepsilon}=\frac{1}{\sqrt{2 \varepsilon}}\left[\frac{1-\cos \left(\theta_{i}-\theta_{j}\right)}{2 \varepsilon}-1\right]+O\left(\varepsilon^{-2}\right),
$$

which is the one of the Hamiltonian mean-field (HMF) model [15]. It is well known that the HMF model [15] has a second order phase transition, separating a low energy phase, where the particles form a single cluster, from a high energy gas phase where kinetic energy dominates and the particles are homogeneously distributed on the circle. One usually draws the so-called caloric curve, where temperature, given by twice the averaged kinetic energy per particle
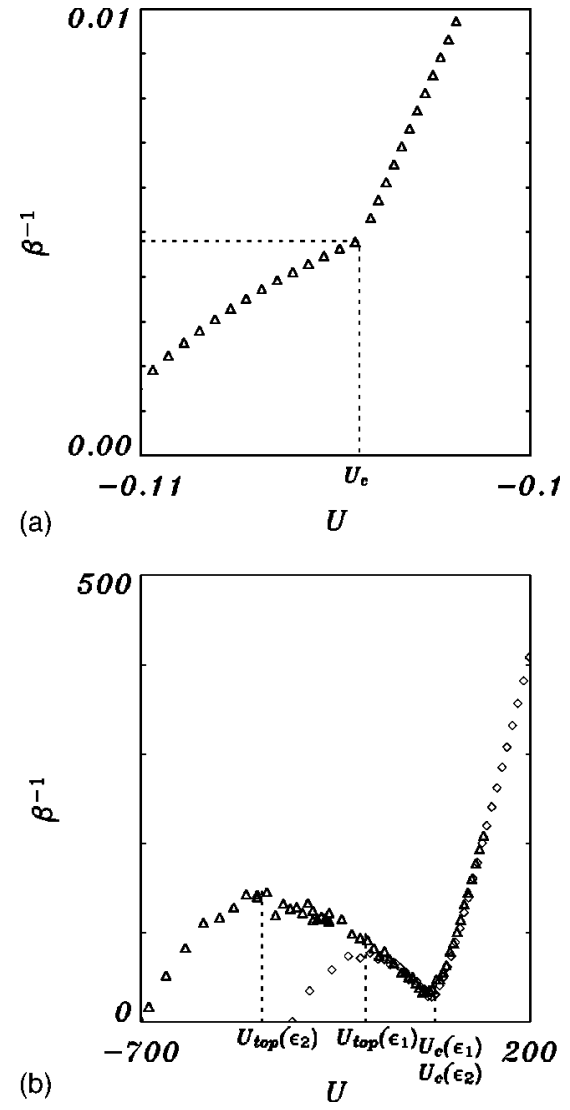

FIG. 2. Caloric curves of the self-gravitating ring (SGR) model obtained from numerical simulations of Hamiltonian (1). Panel (a) refers to the softening parameter value $\varepsilon=10$, for which a second order phase transition appears at $U_{c}$. No backbending of the caloric curve, indicating a negative specific heat, is present. Simulations were performed for $N=100$. Panel (b) shows the caloric curves for two different values of the softening parameter, $\varepsilon_{1}=1.0 \times 10^{-6}$ and $\varepsilon_{2}=2.5 \times 10^{-7}$, and $N=100$. The transition is here first order in the microcanonical ensemble (see Sec. VI for a discussion). The two transition energies $U_{c}\left(\varepsilon_{1}\right)$ and $U_{c}\left(\varepsilon_{2}\right)$ are pretty close, suggesting a slow variation of the critical energy with the softening parameter $\varepsilon$. On the contrary, $U_{\text {top }}\left(\varepsilon_{1}\right)$ is significantly smaller than $U_{\text {top }}\left(\varepsilon_{2}\right)$, indicating that this characteristic energy value diminishes with $\varepsilon$. A negative specific heat phase appears for $U_{\text {top }}<U<U_{c}$, and expands as the softening parameter is reduced. All quantities are plotted in arbitrary units.

$T \equiv \beta^{-1}=2\langle K\rangle / N$, is plotted against the total energy per particle $U \equiv H / N$. In a situation close to that of the HMF model, e.g., for $\varepsilon=10$, the caloric curve determined from microcanonical numerical simulations is reported in Fig. 2(a). In the homogeneous phase $U>U_{c}(\varepsilon)$, the caloric curve is almost linear, while in the clustered phase $U<U_{c}(\varepsilon)$, it is bent downward. Nonetheless, temperature always grows with energy and one does not observe any negative specific heat energy range. However, as it happens for 3D Newtonian gravity simulations [8], when one reduces the softening parameter, a negative specific heat phase develops. For instance, in Fig. 2(b), we show two cases at small $\varepsilon$ where three phases can be identified [14]:

(1) a low-energy clustered phase for $U<U_{\text {top }}(\varepsilon)$, where $U_{\text {top }}$ is defined as the energy at which $\partial T / \partial U=0$. 
(2) an intermediate-energy phase, $U_{\text {top }}(\varepsilon)<U<U_{c}(\varepsilon)$, with negative specific heat.

(3) a high-energy gaseous phase for $U_{c}(\varepsilon)<U$.

The clustered phase is created by the presence of softening $\varepsilon$, without which the particles would fall into the zero distance singularity. In the gas phase, the particles are hardly affected by the potential and behave as almost free particles. The intermediate phase is expected to show the characters of gravity, persisting and even widening in the $\varepsilon \rightarrow 0$ limit.

In the following, several of these features will be given a theoretical explanation and we will detail the analysis of the nature of the phase transition (first or second order) when $\varepsilon$ is varied.

\section{STATIONARY DENSITY DISTRIBUTION}

In the mean-field limit $(N \rightarrow \infty$ with fixed length [20]), one can introduce the single particle distribution function $f(p, \theta)$ such that $f(p, \theta) d p d \theta$ is the fraction of particles in the domain $[\theta, \theta+d \theta][p, p+d p]$. In terms of $f$, the potential energy can be written as

$$
\begin{aligned}
E_{P}[f] & =\frac{1}{2} \int d \theta d \phi d p d p^{\prime} f(p, \theta) V_{\varepsilon}(\theta-\phi) f\left(p^{\prime}, \phi\right) \\
& =\frac{1}{2} \int d \phi d \theta \rho(\theta) \rho(\phi) V_{\varepsilon}(\theta-\phi),
\end{aligned}
$$

where

$$
\rho(\theta)=\int d p f(p, \theta)
$$

is the mass density. The kinetic energy is

$$
E_{K}[f]=\frac{1}{2} \int d \theta d p p^{2} f(p, \theta)
$$

and the total energy

$$
E[f]=E_{K}[f]+E_{P}[f] .
$$

The equilibrium distribution in the microcanonical ensemble is determined by maximizing entropy

$$
S[f]=-\int d \theta d p f \log f
$$

under the constraints of fixed total energy, momentum and mass. In the following, we fix the total energy $E[f]=U$, the total mass

$$
M[f]=\int \rho d \theta=1
$$

and the total momentum

$$
p[f]=\int p f(p, \theta) d \theta d p=0 .
$$

A necessary condition to get an entropy maximum is to require that the free energy

$$
F[f] \equiv S[f]-\beta E[f]-\alpha \int f d p d \theta-\gamma p[f],
$$

where $\alpha, \beta$, and $\gamma$ are Lagrange multipliers, is stationary

$$
\frac{\delta F[f]}{\delta f}=-\log f-1-\beta\left(\frac{p^{2}}{2}+W(\theta)\right)-\alpha-\gamma p=0,
$$

where $W(\theta)$ is defined as

$$
W(\theta) \equiv \int_{-\pi}^{+\pi} \rho(\phi) V_{\varepsilon}(\theta-\phi) d \phi .
$$

Since $p[f]=0$, the Lagrange multiplier $\gamma$ vanishes. From Eq. (13), the normalized stationary distribution function can be written as

$$
f(p, \theta)=A \exp \left[-\beta\left(\frac{p^{2}}{2}+W(\theta)\right)\right],
$$

where $A=\exp (-1-\alpha)$ is the normalization constant and the mass density is given by

$$
\rho(\theta)=\tilde{A} e^{-\beta W(\theta)},
$$

where $\widetilde{A}=A \sqrt{2 \pi / \beta}$. When Eqs. (14) and (16) are combined, we obtain the consistency equations

$$
W(\theta)=\tilde{A} \int_{-\pi}^{+\pi} e^{-\beta W(\phi)} V_{\varepsilon}(\theta-\phi) d \phi,
$$

and the equilibrium density equation

$$
\rho(\theta)=\tilde{A} \exp \left[-\beta \tilde{A} \int_{-\pi}^{+\pi} \rho(\phi) V_{\varepsilon}(\theta-\phi) d \phi\right],
$$

which are solved numerically in the following. Once the stationary mass distributions $\rho$ and the function $W$ are obtained for each value of $\varepsilon$, the full single particle distribution function $f(\theta, p)$ is derived from Eq. (15). The potential energy and the kinetic energy are determined by Eq. (5) and Eq. (7), respectively, allowing to draw the caloric curve by plotting $T \equiv \beta^{-1}=2 E_{K}$ against the total energy $U=E_{K}+E_{P}$.

\section{AN ITERATIVE METHOD TO SOLVE THE EQUILIBRIUM DENSITY EQUATION}

The inverse temperature $\beta$ can be expressed in terms of the energy

$$
\beta=\left\{2 U-\int_{-\pi}^{+\pi} \int_{-\pi}^{+\pi} \rho(\theta) \rho(\phi) V_{\varepsilon}(\theta-\phi) d \theta d \phi\right\}^{-1}
$$

Once an initial density distribution $\rho_{0}(\theta)$ is chosen, one can determine an initial inverse temperature $\beta_{0}$ using Eq. (19), and then solve the consistency equation (18) iteratively (as done for instance in Ref. [21]). However, we will follow here a different iterative method, which ensures entropy increase and, hence, convergence of the algorithm. The method is inspired by a similar one used by Turkington and Whittaker 
[18] to compute entropy maxima for two-dimensional turbulence.

The functional to maximize $S[f]$ is strictly concave and we must fix both a linear constraint $M[f]=1$ and a nonlinear one $E[f]=U$. It is this latter nonlinear constraint which makes the variational problem more difficult than usual. The trick to solve this nonlinear problem consists in considering a linearization of the energy constraint around the distribution function resulting from the previous step in the iterative process.

One begins with the normalized distribution $f_{k}$ obtained at the $k$ th step of the algorithm. From that, one can compute the mass density $\rho_{k}$ and the average potential $W_{k}$.

$$
\begin{gathered}
\rho_{k}(\theta)=\int d p f_{k}(p, \theta), \\
W_{k}(\theta)=\int_{-\pi}^{+\pi} d \phi \rho_{k}(\phi) V_{\varepsilon}(\theta-\phi) .
\end{gathered}
$$

The distribution at the next step $f_{k+1}$ will be then determined by solving the following variational problem

$$
\max \left\{S[f]\left|M[f]=1, E\left[f_{k}\right]+\int \frac{\delta E}{\delta f}\right|_{f_{k}}\left(f-f_{k}\right) d p d \theta \leqslant U\right\},
$$

where the functional derivative of the energy is

$$
\left.\frac{\delta E}{\delta f}\right|_{f_{k}}=\frac{p^{2}}{2}+W_{k}(\theta) .
$$

This variational problem has a unique solution $f_{k+1}$, since it corresponds to the maximization of a strictly concave functional with linear constraints.

This iterative process ensures convergence of the entropy. Let us prove it. By using a generalization of the Lagrange multiplier rule for our inequality constrained variational problem $[22,23]$

$$
\left.\frac{\delta S}{\delta f}\right|_{f_{k+1}}=\alpha_{k+1}+\left.\beta_{k+1} \frac{\delta E}{\delta f}\right|_{f_{k}}
$$

with the additional requirement

$$
\beta_{k+1}\left[E\left[f^{k}\right]+\left.\int \frac{\delta E}{\delta f}\right|_{f_{k}}\left(f_{k+1}-f_{k}\right) d p d \theta-U\right]=0,
$$

where $\beta_{k+1} \geqslant 0$ is the multiplier associated with the energy constraint and $\alpha_{k+1}$, the one associated with mass conservation. When solving Eq. (25), we have either $\beta_{k+1}=0$, which removes the energy constraint, or $\beta_{k+1}>0$, and an equality for the linearized energy constraint.

In order to prove convergence of the entropy, let us first prove that the energy functional $E[f]$ is concave. Since the kinetic part is linear in $f$, the second variation of $E[f]$ is

$$
\begin{aligned}
\delta^{2} E & =\int d \phi d \theta \delta \rho(\theta) \delta \rho(\phi) V_{\varepsilon}(\theta-\phi) \\
& =\sum_{k} V_{\varepsilon, k}\left|\delta \rho_{k}\right|^{2},
\end{aligned}
$$

where the second equality is obtained using the Fourier series expansion for both the mass density variation $\delta \rho$ and the potential $V_{\varepsilon}$

$$
\begin{aligned}
& \delta \rho_{k}=\frac{1}{2 \pi} \int_{-\pi}^{+\pi} d \varphi \exp (i k \varphi) \delta \rho(\varphi), \\
& V_{\varepsilon, k}=\frac{1}{2 \pi} \int_{-\pi}^{+\pi} d \varphi \exp (i k \varphi) V_{\varepsilon}(\varphi) .
\end{aligned}
$$

Since $V_{\varepsilon}$ is even in the argument $\phi, V_{\varepsilon, k}$ is a real number. Moreover, since $V_{\varepsilon} \leqslant 0$ and $V_{\varepsilon}(\varphi)$ is strictly increasing for $0 \leqslant \varphi \leqslant \pi$, it is easy to prove that for any $k, V_{\varepsilon, k}$ is strictly negative. Hence, from formula (27) the second variation of the energy functional is negative and this functional is strictly concave.

On the other hand, the entropy is strictly concave. We have

$$
S[f+\delta f] \leqslant S[f]+\left.\int d \theta d p \frac{\delta S}{\delta f}\right|_{f} \delta f-\frac{1}{2} \int d \theta d p \frac{(\delta f)^{2}}{f},
$$

where in the derivation we have used $\ln (1+x) \geqslant x-x^{2} / 2$ for $x>-1$. Applying this latter inequality with $f=f_{k+1}$ and $\delta f$ $=f_{k}-f_{k+1}$, and using both conditions (24) and (25), we obtain

$$
S\left[f_{k+1}\right]-S\left[f_{k}\right] \geqslant \beta_{k+1}\left(U-E\left[f_{k}\right]\right)+\frac{1}{2} \int d \theta d p \frac{\left(f_{k+1}-f_{k}\right)^{2}}{f_{k}},
$$

where the term proportional to $\alpha_{k+1}$ vanishes because of mass conservation.

We will now use the concavity of the energy functional $E[f]$. For $k>1$,

$$
E\left[f_{k}\right] \leqslant E\left[f_{k-1}\right]+\left.\int \frac{\delta E}{\delta f}\right|_{f_{k-1}}\left(f_{k}-f_{k-1}\right) d p d \theta .
$$

As $\beta_{k+1} \geqslant 0$ and $E\left[f_{k}\right] \leqslant U$, directly from the variational problem (22), Eq. (31) implies that

$$
S\left[f_{k+1}\right]-S\left[f_{k}\right] \geqslant \frac{1}{2} \int \frac{\left(f_{k+1}-f_{k}\right)^{2}}{f_{k}} d \theta d p \geqslant 0 .
$$

Hence, the entropy has to increase for all iterates after the second. Since the entropy is bounded from above, it has to converge. Using Eqs. (31) and (33), one derives that the energy $E\left[f_{k}\right]$ converges to $U$ from below. Moreover, assuming that $f_{k}$ converges toward $f$, one can prove the convergence of the multipliers to limit values $\alpha$ and $\beta \geqslant 0$, which implies that $f$ verifies Eq. (24) for equilibrium states. Although mathematically one cannot prove the convergence of $f$, in all practical cases we will analyze, it appears to be 
verified. For a more thorough discussion of the convergence in the similar case of the Euler equation, see Sec. IV in Ref. [18].

\section{IMPLEMENTATION OF THE ALGORITHM}

We describe in this section the practical implementation of an algorithm which allows the calculation of the stable distribution, using the method described in the previous section.

From (24), we obtain

$$
f_{k+1}=A_{k+1} \exp \left[-\beta_{k+1}\left(\frac{p^{2}}{2}+W_{k}(\theta)\right)\right],
$$

where $A_{k+1}=\exp \left(-\alpha_{k+1}-1\right)$ and $\beta_{k+1}$ are unknown at this stage. Using (20), we get

$$
\rho_{k+1}(\theta)=\widetilde{A}_{k+1} e^{-\beta_{k+1} W_{k}(\theta)},
$$

where $\widetilde{A}_{k+1}=A_{k+1} \sqrt{2 \pi / \beta_{k+1}}$. This equation allows us to compute $W_{k+1}(\theta)$ from Eq. (21) and

$$
E_{k+1} \equiv E\left[f_{k+1}\right]=\frac{1}{2 \beta_{k+1}}+\frac{1}{2} \int_{-\pi}^{+\pi} \rho_{k+1}(\theta) W_{k+1}(\theta) d \theta .
$$

Then the multipliers $\alpha_{k+1}$ and $\beta_{k+1}$ must be computed from Eqs. (10) and (25) and, from these, one gets $\widetilde{A}_{k+1}$. In order to compute numerically these Lagrange parameters, let us define the Lagrangian [23]

$$
\begin{aligned}
L_{k}[f](\beta, \alpha)= & -S[f]+\beta\left[E_{k}+\left.\int \frac{\delta E}{\delta f}\right|_{f_{k}}\left(f-f_{k}\right) d p d \theta-U\right] \\
& +\alpha(M[f]-1) .
\end{aligned}
$$

From this, one further defines

$$
L_{k}^{\star}(\beta, \alpha)=\inf _{f}\left\{L_{k}[f](\beta, \alpha)\right\} .
$$

One can prove on a general ground [23] that $L_{k}^{\star}$ is concave and that $\alpha_{k+1}$ and $\beta_{k+1}$ are the unique maxima of $L_{k}^{\star}$. Using condition (34) for the extrema of $L_{k}[f](\beta, \alpha)$, we can compute $L_{k}^{\star}$. We obtain, using for practical reasons the variable $\tilde{A}$ instead of $\alpha$,

$$
\begin{aligned}
L_{k}^{\star}(\beta, \tilde{A})= & \log \tilde{A}+\frac{1}{2} \log \beta-\beta\left(U+E_{k}-\frac{1}{2 \beta_{k}}\right) \\
& -\tilde{A} \int_{-\pi}^{+\pi} d \theta e^{-\beta W_{k}(\theta)} .
\end{aligned}
$$

Necessary conditions for the concave function $L_{k}^{\star}$ to be maximal are

$$
\frac{\partial L_{k}^{\star}}{\partial \tilde{A}}=\frac{1}{\tilde{A}}-\int_{-\pi}^{+\pi} d \theta e^{-\beta W_{k}(\theta)}=0,
$$

$$
\frac{\partial L_{k}^{\star}}{\partial \beta}=\frac{1}{2 \beta}+\frac{1}{2 \beta_{k}}-U-E_{k}+\tilde{A} \int_{-\pi}^{+\pi} d \theta W_{k}(\theta) e^{-\beta W_{k}(\theta)}=0 .
$$

Substituting Eq. (40) into Eq. (41), one gets the condition

$$
-\frac{1}{2 \beta_{k+1}}-\frac{1}{2 \beta_{k}}+U+E_{k}-\frac{\int_{-\pi}^{+\pi} d \theta W_{k}(\theta) e^{-\beta_{k+1} W_{k}(\theta)}}{\int_{-\pi}^{+\pi} d \theta e^{-\beta_{k+1} W_{k}(\theta)}}=0,
$$

which, since $L_{k}^{\star}$ is concave, has a unique solution. Numerically, the solution $\beta_{k+1}$ is found by using a Newton algorithm for Eq. (42). Then, from Eq. (40), we get $\widetilde{A}_{k+1}$. Finally, we can calculate the density distribution from Eq. (35).

\section{DISCUSSION OF THE RESULTS}

Using the iterative method described in the previous section, we are able to derive the stable mass density $\rho(\theta)$ solution of Eq. (18) and, from that, all thermodynamic functions in the microcanonical ensemble. In the first part of this section, we will show the numerical solution obtained for $\rho(\theta)$, and its dependence on energy for a small value of the softening parameter $\varepsilon$. In the second part, we will discuss the phase diagram of the SGR model, both in the microcanonical and in the canonical ensemble, when $\varepsilon$ is varied.

\section{A. Mass density, entropy and caloric curves}

For energies above a certain critical value $U_{c}(\varepsilon)$, the stable mass density solution is uniform. In this case, one can compute the entropy from Eq. (9)

$$
S=\frac{1}{2}[3 \log (2 \pi)+1-\log \beta],
$$

and the inverse temperature from Eq. (19)

$$
\beta=\left(2 U-2 \bar{E}_{p}\right)^{-1},
$$

where

$$
\begin{aligned}
\bar{E}_{p} & =\frac{1}{2} \frac{1}{(2 \pi)^{2}} \int_{-\pi}^{+\pi} \int_{-\pi}^{+\pi} d \theta d \phi V_{\varepsilon}(\theta-\phi) \\
& =-\frac{1}{\pi \sqrt{2}} \frac{1}{\sqrt{2+\varepsilon}} \mathcal{K}\left(\frac{2}{\varepsilon+2}\right),
\end{aligned}
$$

where $\mathcal{K}$ is the complete elliptic integral of the first kind $\mathcal{K}(x) \equiv \int_{0}^{\pi / 2} d \theta / \sqrt{1-x \sin ^{2} \theta}$.

Remark that Eq. (44) implies that the homogeneous state cannot be continued below $U_{\text {hom }}=\bar{E}_{p}$, because this latter energy corresponds to zero temperature.

For $U<U_{c}(\varepsilon)$, the stable mass distribution must be determined numerically. We have checked in this case, that a direct iterative method of solution of the consistency Eqs. (17) and (18) does not always converge. On the contrary, the algorithm presented in Sec. V ensures convergence as shown in Fig. 3 for the entropy. 


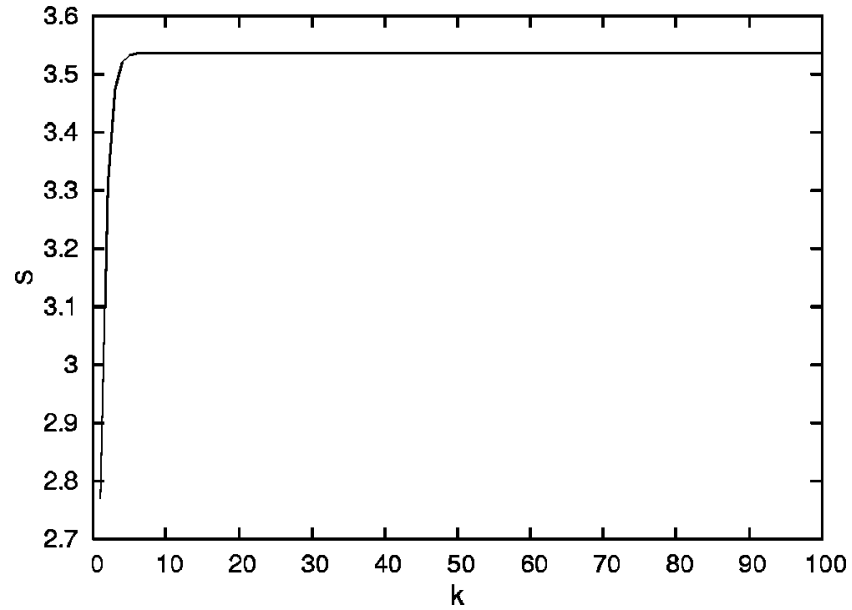

FIG. 3. Convergence of the entropy using the algorithm of Sec. $\mathrm{V}$ for $\varepsilon=10^{-5}$ and $U=-1$. All quantities are plotted in arbitrary units.

In Fig. 4 we show both entropy and temperature $T=\beta^{-1}$ as a function of energy $U$. The most striking feature is the presence of a negative specific heat region for $U_{t o p} \leqslant U \leqslant U_{c}$. For $U_{\text {low }} \leqslant U \leqslant U_{\text {high }}$, the entropy does not coincide with its convex envelope. Hence, microcanonical and canonical ensembles do not give the same predictions. Indeed, the main peculiarity of the microcanonical ensemble is that macroscopic states within this interval are stable, while they would be either metastable or unstable in the canonical ensemble. The mass density is uniform above $U_{c}$, while, below this value, it is localized. The appropriate order parameter to characterize this localization is the "magnetization"

$$
B=\int_{-\pi}^{+\pi} d \theta e^{i \theta} \rho(\theta),
$$

which vanishes if the mass distribution is uniform while it reaches the value $B=1$ when the mass is concentrated in only one point. Intermediate degrees of localization give intermediate values of $B$. The "magnetization" is plotted in Fig. 5 as a function of $U$. It is a decreasing function of $U$, up to $U_{c}$, where it has a jump to the limiting value 0 . Hence, we have here a first order microcanonical phase transition. The first order nature of the phase transition is confirmed zooming the entropy around $U_{c}$ [see the inset in panel (b) of Fig. 4]. This reveals that this first order phase transition is of the convexconcave type (see Ref. [17]). The canonical ensemble is obtained by taking the convex envelope of the microcanonical entropy. The transition is first order in the canonical ensemble and the transition temperature $T_{c a n}$ is given by the inverse slope of the entropy at $U_{\text {low }}$ and $U_{\text {high }}$. No canonical macrostate is present in the energy range $\left[U_{\text {low }}, U_{\text {high }}\right]$.

A typical localized mass density distribution is shown in Fig. 6. It corresponds to an energy where the specific heat is negative.

The first order phase transition is associated with the existence of metastable states. Using a continuation method, we have been able to compute them. Their entropy is represented in Fig. 7 around the transition energy $U_{c}$ for the spe-
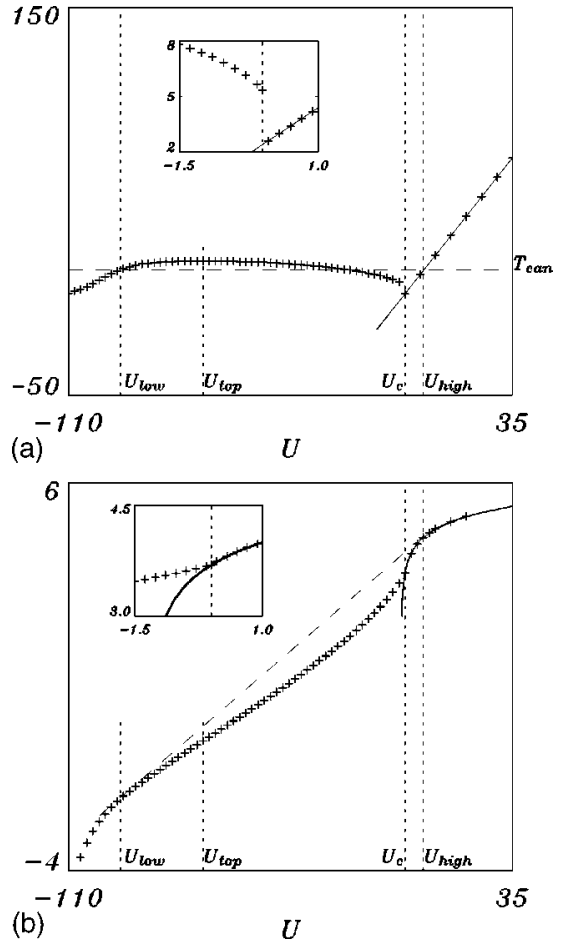

FIG. 4. Temperature [panel (a)] and entropy [panel (b)] versus energy $U$ for the softening parameter value $\varepsilon=10^{-5}$. Four values of the energy, indicated by the short-dashed vertical lines, can be identified from this picture: $U_{\text {low }} \simeq-93$ and $U_{\text {high }} \simeq 6$ bound from below and above the region of inequivalence of ensembles. $U_{c} \simeq 0$ is the transition energy in the microcanonical ensemble. $U_{\text {top }} \simeq-66$ limits from below the negative specific heat region, where temperature decreases as energy increases. $T_{c a n} \simeq 15$, represented with a dashed line in panel (a), is the canonical transition temperature and corresponds to the inverse slope of the entropy, both at $U_{\text {low }}$ and $U_{\text {high }}$, as represented by the straight dashed line in panel (b). The full lines represent the analytical solutions of the temperature and of entropy in the uniform case [see formulas (43) and (44)]. They are extended slightly below $U_{c}$, in the metastable phase, in order to identify them. The insets in panels (a) and (b) show a zoom of the temperature and of the entropy around $U_{c}$, revealing a temperature jump at $U_{c}$ and different slopes of the entropy above and below $U_{c}$, which emphasizes the first order nature of the phase transition. All quantities are plotted in arbitrary units.

cific case $\varepsilon=10^{-5}$. The inhomogeneous metastable state turns out to exist for $U_{c} \leqslant U \leqslant U_{\text {in }}$ with $U_{\text {in }} \simeq 0.16$, while the homogeneous metastable state exists for $U_{\text {hom }} \leqslant U \leqslant U_{c}$, with $U_{\text {hom }}=\bar{E}_{p}\left(\varepsilon=10^{-5}\right) \simeq-1.19$.

\section{B. Behavior as the softening parameter $\varepsilon$ is varied}

Let us first examine a situation where the softening parameter is much larger than previously, $\varepsilon=10^{-2}$. In the microcanonical ensemble, Fig. 8 shows that a concavity change still occurs at $U_{\text {top }} \simeq-0.8$, and that a phase transition exists at $U=U_{c} \simeq-0.3$. However, the temperature being now a continuous function of the energy but with discontinuous derivative at $U_{c}$, the phase transition is of second order (see Fig. 9), and is associated with the symmetry breaking of the order 


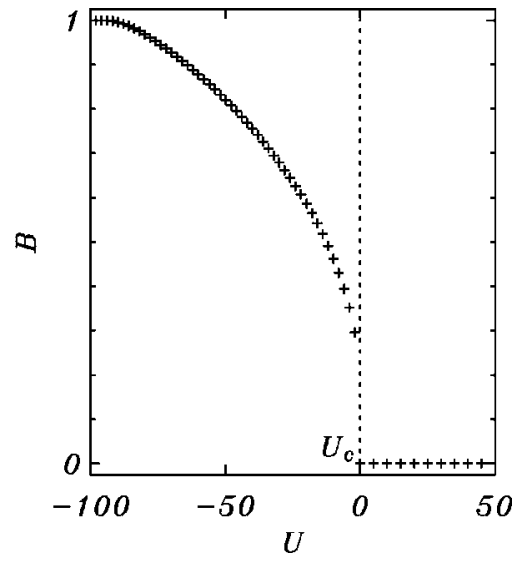

FIG. 5. "Magnetization" $B$ versus energy $U$ for $\varepsilon=10^{-5}$, which emphasizes the microcanonical first order phase transition at $U_{c}$ $\simeq 0$ by showing a jump in the order parameter. All quantities are plotted in arbitrary units.

parameter. The caloric curve shows that this second order phase transition is of the convex-concave type. As it is necessary for this type of microcanonical second order phase transition [17], we observe a positive specific heat jump at the transition point.

What we find suggests that between $\varepsilon=10^{-5}$ and $\varepsilon=10^{-2}$, there is an intermediate value of $\varepsilon$ where a microcanonical tricritical point is present. This point is signaled by two properties:

(1) The caloric curve assumes a negative infinite slope as $U$ tends to $U_{c}$ from below.

(2) The upper energy of the metastable inhomogeneous phase $U_{\text {in }}$ collapses onto $U_{c}$ from above, while still a continuation of the homogeneous phase below $U_{c}$ exists as an unstable phase.

In Fig. 10, we have represented the $\varepsilon$ dependence of the critical energy $U_{c}$ and of the energy bounds $U_{\text {in }}$ and $U_{\text {hom }}$. At the microcanonical tricritical point, $\varepsilon_{T}^{\mu} \simeq 10^{-4}$, the end point for the existence of the inhomogeneous metastable phase

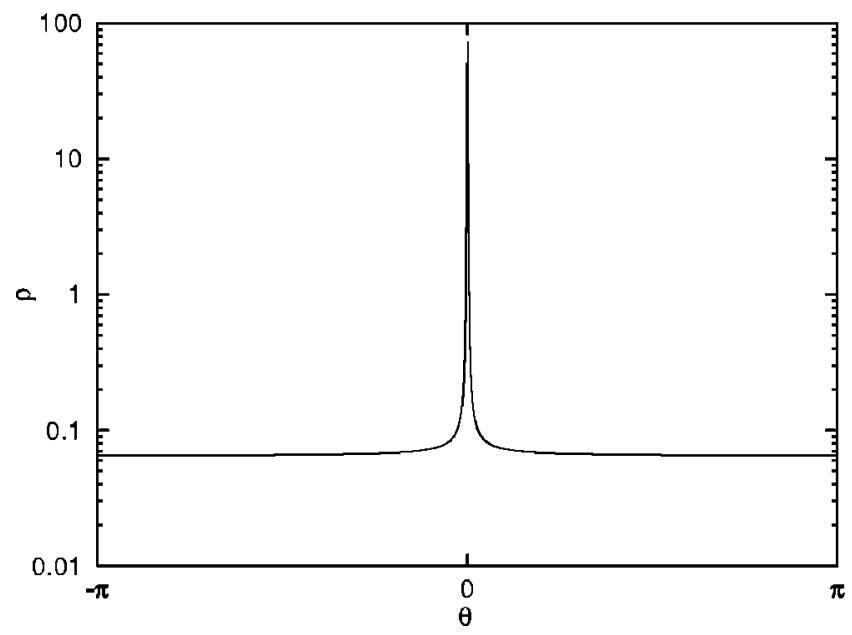

FIG. 6. A typical mass density distribution $\rho(\theta)$ for $\varepsilon=10^{-5}$ and $U=-20.0$, in the negative specific heat region. All quantities are plotted in arbitrary units.

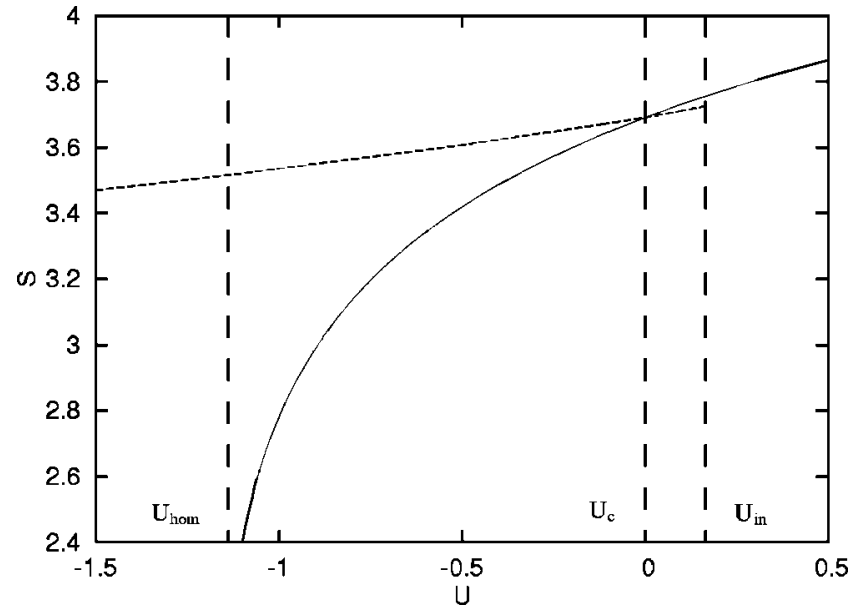

FIG. 7. Both the high energy branch of the entropy versus energy curve, corresponding to the homogeneous solution (solid line) and the low energy branch of the inhomogeneous solution (dashed line) are represented in this plot for $\varepsilon=10^{-5}$. The two branches cross at $U_{c} \simeq 0$. The continuation of the homogeneous branch into the low energy region is bounded from below by $U_{\text {hom }} \simeq-1.19$, indicated by a vertical dashed line. The inhomogeneous branch continues into the high energy phase and ends at $U_{i n} \simeq 0.16$, again indicated by a vertical dashed line. All quantities are plotted in arbitrary units.

joins the critical line. This is a generic feature of tricritical points with symmetry breaking (see Fig. 6 of Ref. [17]).

To locate the tricritical point in the canonical ensemble, one has to look for the $\varepsilon$ value at which the two curves $U_{\text {low }}$ and $U_{\text {high }}$ merge (see Fig. 11). An approximate estimate of this value is $\varepsilon_{T}^{c} \simeq 10^{-1}$. At the canonical tricritical point, also $U_{\text {top }}$ merges with the above curves, indicating the disappearance of the negative specific heat region. We thus note that ensemble inequivalence disappears at the canonical tricritical point by the disappearance of the inflection point at $U_{\text {top }}$ in the entropy curve. As it may be checked in Table I of Ref. [17], this is the only way in which ensemble inequivalence can disappear when associated with a tricritical point.

Summarizing, the important changes of the phase diagram of the SGR model when $\varepsilon$ is varied are due to the existence of microcanonical and canonical tricritical points. For $\varepsilon$ $\leqslant \varepsilon_{T}^{c}$, there is an energy range with ensemble inequivalence. These features were already observed in Refs. [19,24].

\section{RELAXATION TO EQUILIBRIUM}

We have first checked numerically if the equilibrium density profile is ever attained in direct $N$-body simulations of Hamiltonian (1). In Fig. 12, we compare the result of a numerical simulation with the equilibrium density profile obtained by the iterative method. The agreement is good in the center of the mass distribution, while the tails are still affected by strong finite $N$ fluctuations. In this case, the total energy $U=-20$ is in the region of negative specific heat and is well conserved using a sixth order symplectic integrator [25]. Simulations were performed using GRAPE-5, a special purpose computer for gravitational force [9].

However, it is well known that systems with long-range interactions display a very slow relaxation to equilibrium 


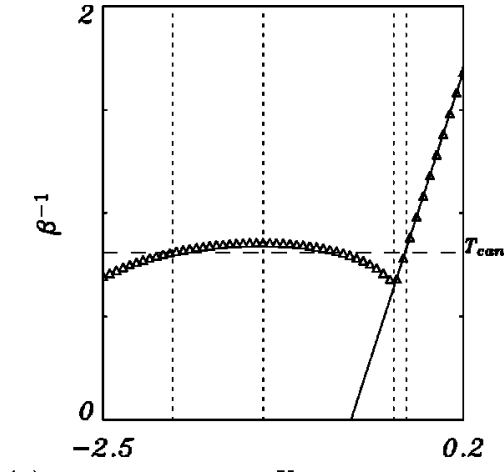

(a)

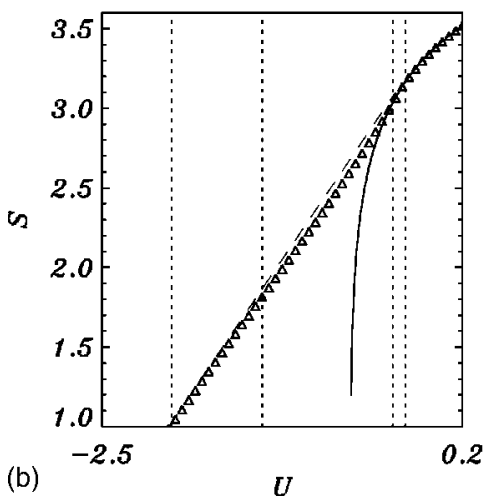

FIG. 8. Panel (a): The caloric curve (triangles) for $\varepsilon=10^{-2}$. The dashed vertical lines indicate, from left to right, $U_{\text {low }} \simeq-1.98$, $U_{\text {top }} \simeq-1.3, U_{c} \simeq-0.32$, and $U_{\text {high }} \simeq-0.225$. The homogeneous phase curve, known analytically, is shown by the continuous line. The main difference with respect to Fig. 4 is that now there is not a temperature jump at $U_{c}$. The phase transition is second order in the microcanonical ensemble, while it is still first order in the canonical ensemble, at $T_{c a n} \simeq 0.8$. Panel (b): Entropy versus energy (triangles) for $\varepsilon=10^{-2}$. The entropy curve corresponding to the inhomogeneous distribution smoothly connects with the one of the homogeneous distribution (solid line). The oblique straight dashed line is tangent to the entropy at $U_{\text {low }}$ and $U_{\text {high }}$, which delimit the energy region of ensemble inequivalence. All quantities are plotted in arbitrary units.

[26]. Hence, we expect that similar features will be also exhibited by the SGR model. For instance, we can consider a "cold start," where the particles are initially homogeneously distributed on an $\operatorname{arch}\left(\theta \in\left[\theta_{\min }, \theta_{\max }\right]\right)$ with zero kinetic energy. Usually, in gravitational simulations, one looks at the evolution of the virial ratio $|2 K / V|$, which is here initially zero. The plot of the time dependence of this parameter is shown in Fig. 13 for the same parameter values used previously. One clearly observes that the system relaxes to a "quasiequilibrium" state, where the virial ratio fluctuates around a value which differs from the equilibrium one, computed analytically. While previously, for the mass positions (see Fig. 12), the relaxation was observed on a short time scale, we show here that a quantity related also to velocities does not display a relaxation on the same time scale. From previous experiences with similar cases [26], one expects that the relaxation should occur on a time scale of the order of a power of $N$.

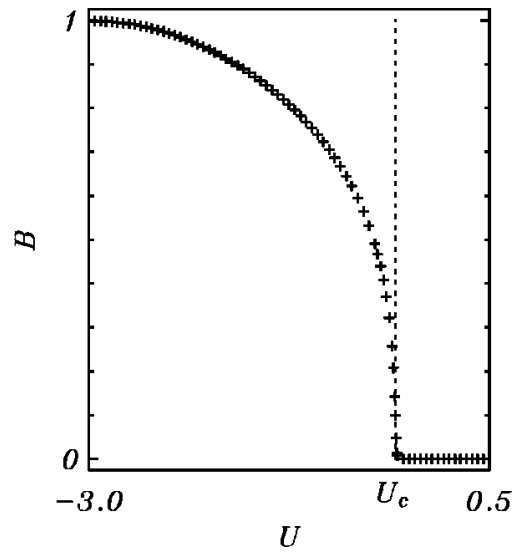

FIG. 9. "Magnetization" $B$ versus energy $U$ for $\varepsilon=10^{-2}$ which emphasizes the microcanonical second order phase transition at $U_{c}$, because the order parameter vanishes continuously. All quantities are plotted in arbitrary units.

Even slower is the relaxation when local maxima of the entropy exist. This happens around the critical energy $U_{c}$ in the case of a first order phase transition, e.g., for $\varepsilon=10^{-5}$. Figure 14 shows the relaxation to different values of two relevant quantities, the temperature and the "magnetization," when the system is initialized either with the particles concentrated on a small arch, or on a larger one. When the system is "close" to the local entropy maximum corresponding to the clustered state, it converges to it pretty fast. The contrary happens when the particles are more homogeneously distributed, and then the system converges to the homogeneous state. Indeed, between the two states there is an entropy barrier which has been found to grow as $\exp (N)$ for systems with long-range interactions $[27,28]$.

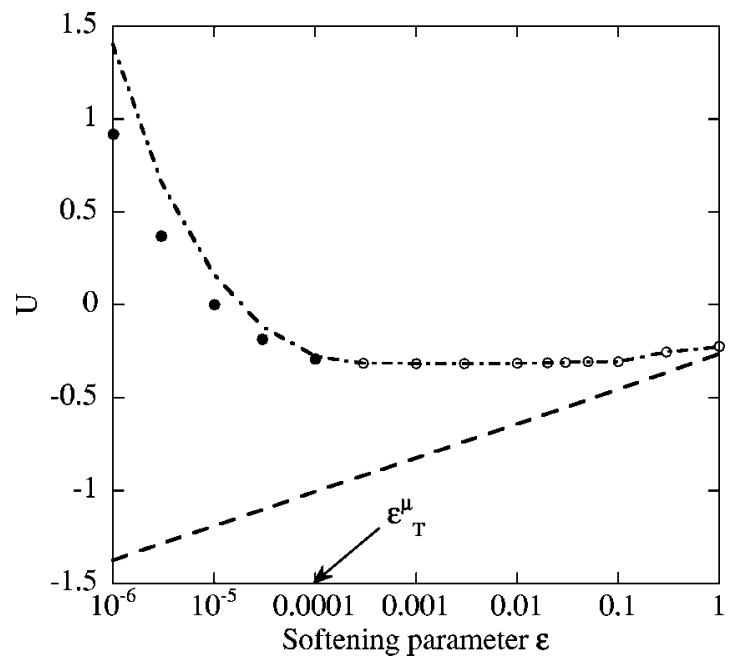

FIG. 10. The dash-dotted line represents $U_{i n}(\varepsilon)$, the dashed line $U_{\text {hom }}=\bar{E}_{p}(\varepsilon)$, the filled circles the first order microcanonical phase transition energy, and the open circles the second order one. At the microcanonical tricritical point $\varepsilon_{T}^{\mu} \simeq 10^{-4}$, the phase transition changes from first order to second order and, at the same time, the inhomogeneous metastable solution disappears. All quantities are plotted in arbitrary units. 


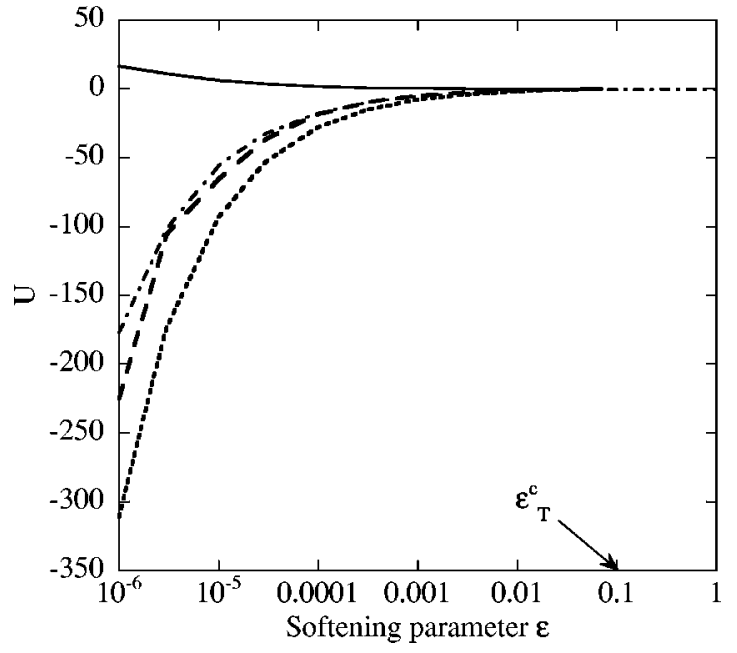

FIG. 11. $\varepsilon$ dependence of $U_{\text {low }}$ (dashed line), $U_{\text {high }}$ (solid line), and $U_{t o p}$ (dash-dotted line). The canonical tricritical point is located at $\varepsilon_{T}^{c} \simeq 10^{-1}$ where the three curves merge. At this softening parameter value also the negative specific heat disappears in the microcanonical ensemble, while the transition becomes second order in the canonical ensemble. In the figure, we also show, with a long-dashed line, the theoretical estimate of $U_{\text {top }}^{\text {th }} \simeq-1 /(4 \sqrt{2 \varepsilon})$ obtained in Ref. [14]. All quantities are plotted in arbitrary units.

\section{CONCLUSIONS AND PERSPECTIVES}

We have fully characterized from the thermodynamic point of view a one-dimensional model of self-gravitating particles moving on a ring [14], which is the simplest prototype of the full 3D self-gravitating system. Solving the equilibrium density equation by an iterative method, whose convergence is assured by entropy increase, allows us to derive the full phase diagram of the model both in the microcanonical and the canonical ensemble. When the softening param-

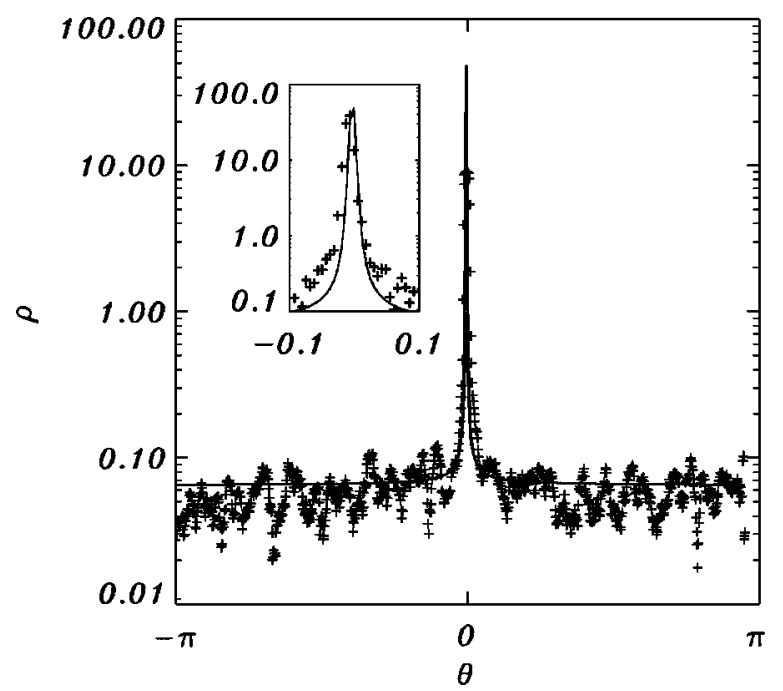

FIG. 12. Comparison of the mass density profile obtained by the iterative method (solid line) with the result of numerical simulations (plus signs) with $N=4000$ and $\varepsilon=10^{-5}$. Parameter values are the same as Fig. 6. The inset is a zoom of the center of the profile. All quantities are plotted in arbitrary units.

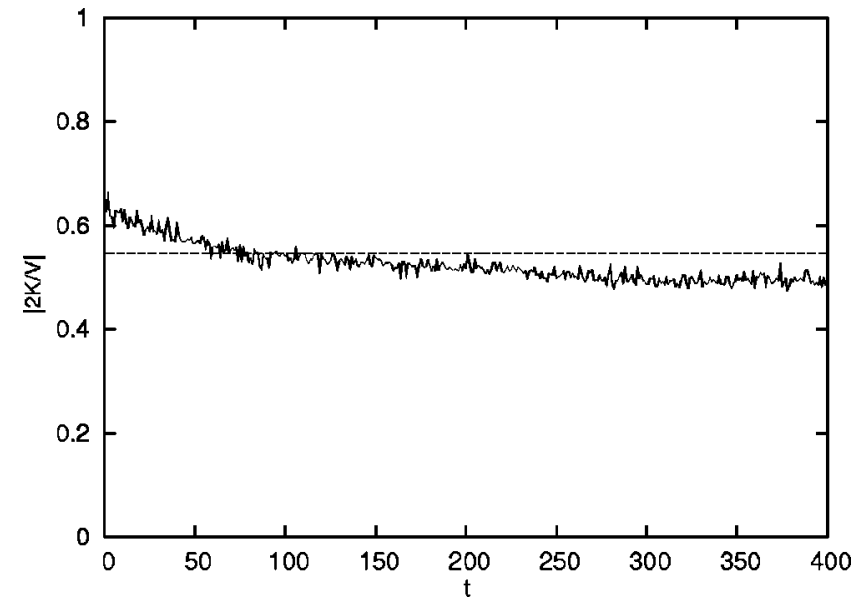

FIG. 13. Time evolution of the virial ratio $|2 K / V|$ of the SGR model for $\varepsilon=10^{-5}$ and $N=4000$. Initially, the particles are homogeneously distributed in the interval $[0,2 \pi / 75]$ with zero kinetic energy. The virial ratio oscillates asymptotically around the value 0.49 , which differs significantly from the equilibrium value 0.55 indicated by the dashed horizontal line. The initial virial ratio is zero, although this time region is not visible in the figure. All quantities are plotted in arbitrary units.

eter is sufficiently small, a negative specific heat region appears in the microcanonical ensemble, in coincidence with the phase transition becoming first order in the canonical ensemble. Further lowering the softening parameter, the transition becomes first order in the microcanonical ensemble and a temperature jump appears at the transition energy. The microcanonical and canonical tricritical points do not coincide [19].

Dynamically, we have performed numerical experiments which show that relaxation to equilibrium can be extremely slow. They reveal also the presence of quasiequilibrium states, which are ubiquitous in systems with long-range interactions [16]. These states could be further characterized considering a Vlasov equation approach as done for the
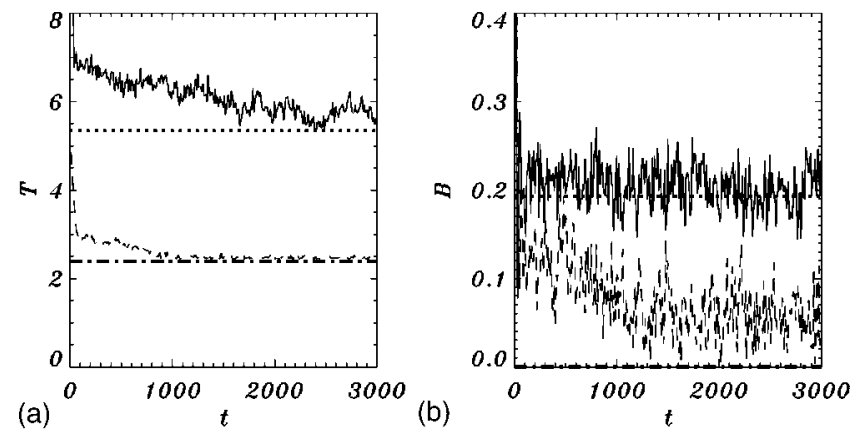

FIG. 14. Relaxation to different maximum entropy states in the SGR model for $U \simeq 0, \varepsilon=10^{-5}$, and $N=10^{3}$. Panel (a) shows the relaxation of the temperature either to the inhomogeneous state value (horizontal dotted line), or to the homogeneous one (horizontal dash-dotted line), depending on whether the particles are initially distributed on a smaller arch $\theta \in[0, \pi / 50]$ (solid line) or a larger arch $\theta \in[0, \pi / 5]$ (dashed line). In both cases, the velocity distribution is initially a "water bag." Panel (b) shows the same for the "magnetization." All quantities are plotted in arbitrary units. 
HMF model in Ref. [26]. Moreover, in the first-order microcanonical transition region a strong metastability appears and, at a given energy, the system can relax towards different thermodynamic states.

Preliminary studies of velocity probability distributions in this model have been performed in Ref. [14]. A similar analysis has been more recently done for the full 3D selfgravitating system [29]. In both models, non-Gaussian tails show up in several energy regions. Among future directions of study of the SGR model, we think that deriving a theoretical framework to undertand these large tails would be of particular interest. To this aim, especially useful could be the methods developed to understand single-particle diffusion in the HMF model [30,31].

\section{ACKNOWLEDGMENTS}

We thank Osamu Iguchi, Kei-ichi Maeda, Masahiro Morikawa, Akika Nakamichi, and Yasuhide Sota for useful discussions. All numerical simulations were carried out on the GRAPE system at the Astronomical Data Analysis Center (ADAC) of the National Astronomical Observatory, Japan. T.T. thanks ENS Lyon for hospitality. S.R. thanks ENS Lyon for hospitality and CNRS for financial support. This work is part of the contract COFIN03 of the Italian MIUR "Order and chaos in nonlinear extended systems," and was partially supported by a Grant-in-Aid for Scientific Research Fund of the Ministry of Education, Culture, Sports, Science and Technology, Japan [Young Scientists (B) 16740152].
[1] J. Binney and S. Tremaine, Galactic Dynamics (Princeton University Press, Princeton, NJ, 1987).

[2] V. A. Antonov, Vestn. Leningr. Univ., Ser. 3: Biol. 7, 135 (1962).

[3] D. Lynden-Bell and R. Wood, Mon. Not. R. Astron. Soc. 138, 495 (1968).

[4] T. Padmanabhan, Astrophys. J., Suppl. Ser. 71, 651 (1989).

[5] P. H. Chavanis, Phys. Rev. E 65, 056123 (2002).

[6] P. H. Chavanis and I. Ispolatov, Phys. Rev. E 66, 036109 (2002).

[7] B. Stahl, M. K.-H. Kiessling, and K. Schindler, Planet. Space Sci. 43, 271 (1995).

[8] D. Heggie and P. Hut, The Gravitational Million-Body Problem (Cambridge University Press, Cambridge, England, 2003).

[9] A. Kawai, T. Fukushige, J. Makino, and M. Taiji, Publ. Astron. Soc. Jpn. 52, 659 (2000).

[10] F. Hohl and M. R. Feix, Astrophys. J. 147, 1164 (1967).

[11] T. Tsuchiya, T. Konishi, and N. Gouda, Phys. Rev. E 50, 2607 (1994).

[12] H. Koyama and T. Konishi, Phys. Lett. A 279, 226 (2001).

[13] V. P. Youngkins and B. Miller, Phys. Rev. E 62, 4583 (2000).

[14] Y. Sota, O. Iguchi, M. Morikawa, T. Tatekawa, and K. Maeda, Phys. Rev. E 64, 056133 (2001).

[15] M. Antoni and S. Ruffo, Phys. Rev. E 52, 2361 (1995).

[16] Dynamics and Thermodynamics of Systems with Long-Range Interactions, edited by T. Dauxois, S. Ruffo, E. Arimondo, and M. Wilkens, Lecture Notes in Physics Vol. 602 (Springer, Ber- lin, 2002).

[17] F. Bouchet and J. Barré, J. Stat. Phys. 118, 1073 (2005).

[18] B. Turkington and N. Whitaker, SIAM J. Sci. Comput. (USA) 17, 1414 (1996).

[19] J. Barré, D. Mukamel, and S. Ruffo, Phys. Rev. Lett. 87, 030601 (2001).

[20] J. Messer and H. Spohn, J. Stat. Phys. 29, 561 (1982).

[21] I. Ispolatov and E. G. D. Cohen, Phys. Rev. Lett. 87, 210601 (2001).

[22] A. D. Ioffe and V. M. Tikhomirov, Theory of Extremal Problems (Elsevier-North-Holland, New York, 1979).

[23] R. T. Rockafellar, Convex Analysis (Princeton University Press, Princeton, NJ, 1970).

[24] M. Antoni, S. Ruffo, and A. Torcini, Phys. Rev. E 66, 025103 (2002).

[25] H. Yoshida, Phys. Lett. A 150, 262 (1990).

[26] Y. Y. Yamaguchi, J. Barré, F. Bouchet, T. Dauxois, and S. Ruffo, Physica A 337, 36 (2004).

[27] M. Antoni, S. Ruffo, and A. Torcini, Europhys. Lett. 66, 645 (2004).

[28] P. H. Chavanis and M. Rieutord, Astron. Astrophys. 412, 1 (2003); P. H. Chavanis, astro-ph/0404251.

[29] O. Iguchi, Y. Sota, T. Tatekawa, A. Nakamichi, and M. Morikawa, Phys. Rev. E 71, 016102 (2005).

[30] F. Bouchet, Phys. Rev. E 70, 036113 (2004).

[31] F. Bouchet and T. Dauxois, cond-mat/0407703. 